\title{
Study of the introduction collection of the Miscanthus
}

Kapustyanchik S.Iu. ${ }^{1}$, Kapko T.N. ${ }^{1}$, Totsky I.V. ${ }^{1}$, Khlestkina E.K. ${ }^{1,2}$, Potseluev O.M. ${ }^{1}$

${ }^{1}$ Institute of Cytology and Genetics, SB RAS, Novosibirsk, Russia

${ }^{2}$ N.I. Vavilov All-Russian Institute of Plant Genetic Resources (VIR), St. Petersburg, Russia

*e-mail: kapustyanchik@bionet.nsc.ru

Currently, the most accurate methods for establishing variety identity are DNA polymorphism analysis methods. One of such methods widely used for certification is microsatellite analysis. Microsatellite markers developed for Miscanthus have not yet been introduced into practical application for the certification of varieties. The aim of the current study was to develop a method of genetic certification of samples of the Miscanthus collection based on microsatellite analysis. A total of 47 Miscanthus samples were studied: 23 accessions from the introduction collection (21 accessions of M. sacchariflorus, 1 hybrid of M. giganteus and 1 hybrid sample, which taxonomic assignment is still to be clarified) and 24 plant samples of M. sacchariflorus cv. Soranovsky, having different morphotypes. The latter was included to study intravarietal polymorphism of the cv. Soranovsky to check whether there are genetic differences between different ecotypes. When selecting plants cv. Soranovsky, attention was paid to such signs as the presence (plants no. 1-7) and absence (plants no. 9-16) of a full panicle, as well as a tendency to lodging (plants no. 17-24). A total of 10 primer pairs for amplification of highly polymorphic microsatellite loci were selected from the literature. Based on the cross-assessment of these primers for the formation of dimers, the optimal combinations for multiplex PCR were selected. The PCR testing was carried out on individual primer pairs on the extracted DNA. Three sets of fluorescently labeled markers were designed for further multiplex PCR and fragment analysis. Identification of various samples of Miscanthus, as well as evaluation of intraspecific polymorphism of the cv. Soranovsky, is carried out. 